\title{
Synthesis, characterization and antimicrobial evaluation of the novel pyrido[1',2':2,3][1,2,4]triazolo[1,5-c]quinazolines and thiazolo $\left[3^{\prime}, 2^{\prime}: 2,3\right][1,2,4]$ triazolo $[1,5-a]$ pyridines
}

\author{
Azza M. El-Kazak and Magdy A. Ibrahim* \\ Department of Chemistry, Faculty of Education, Ain Shams University, \\ Roxy, 11711, Cairo-Egypt \\ E-mail: magdy_ahmed1977@yahoo.com
}

\begin{abstract}
Starting from ethyl 1,2-diamino-5-cyano-4-(4-methoxyphenyl)-6-oxo-1,6-dihydropyridine-3carboxylate, a novel series of polynuclear pyrido[1',2':2,3][1,2,4]triazolo[1,5-c]quinazolines and thiazolo[3',2':2,3][1,2,4]triazolo[1,5-a]pyridines have been synthesized. Structures of the newly synthesized products have been deduced on the basis of elemental analysis and spectral data. The synthesized compounds were screened for their antimicrobial activity.
\end{abstract}

Keywords: $\quad$ 1,2-Diaminopyridone, $\quad$ pyrido[1',2':2,3][1,2,4]triazolo[1,5-c]quinazolines, thiazolo[3',2':2,3][1,2,4]triazolo[1,5-a]pyridines, cyclocondensation

\section{Introduction}

$o$-Diamines are very active substrates for building of various heterocyclic systems.$^{1-7}$ 4-Aryl-1,6diamino-2-oxo-1,2-dihydropyridine-3,5-dicarbonitriles have been used for the synthesis of biologically active nitrogen bridgehead triazolo[1,5-a]pyridines, ${ }^{8-10}$ pyrido[1,2- $b$ ] $[1,2,4]$ triazines, ${ }^{11-13}$ and pyrido[1,2-b][1,2,4]triazepines. ${ }^{14,15}$ The present work aimed to utilize the diaminopyridone derivative $\mathbf{2}$ as a precursor to synthesis a novel series of polyheterocyclic systems namely pyrido[1',2':2,3][1,2,4]triazolo[1,5-c]quinazolines and thiazolo[3',2':2,3][1,2,4] triazolo[1,5-a]pyridines to evaluate their antimicrobial activity.

\section{Results and Discussion}

The starting compound ethyl 1,2-diamino-5-cyano-4-(4-methoxyphenyl)-6-oxo-1,6dihydropyridine-3-carboxylate (2) was prepared by refluxing an alcoholic solution of ethyl 3cyano-2-(4-methoxyphenyl)prop-2-enoate (1) with cyanoacetohydrazide in absolute ethanol 
containing few drops of piperidine as a catalyst (Scheme 1). ${ }^{16}$ The IR spectrum of compound 2 showed characteristic absorption bands at 3216, 3089, $3079\left(2 \mathrm{NH}_{2}\right), 2255(\mathrm{C} \equiv \mathrm{N}), 1692$ $\left(\mathrm{C}=\mathrm{O}_{\text {ester }}\right)$ and $1672 \mathrm{~cm}^{-1}\left(\mathrm{C}=\mathrm{O}_{\text {pyridone }}\right)$. Its mass spectrum revealed the molecular ion peak at $\mathrm{m} / \mathrm{z}$ 328 which agree well with the molecular weight (328.33) as supports the identity of the structure.

Treating compound 2 with anthranilic acid in phosphorus oxychloride gave ethyl 2-(2aminophenyl)-6-cyano-7-(4-methoxyphenyl)-5-oxo-3,5-dihydro[1,2,4]triazolo[1,5-a]pyridine-8carboxylate (3) (Scheme 1). ${ }^{17}$ The IR spectrum of compound $\mathbf{3}$ exhibited characteristic absorption bands at 3417, 3341, $3241\left(\mathrm{NH}_{2}, \mathrm{NH}\right), 2189(\mathrm{C} \equiv \mathrm{N}), 1678\left(\mathrm{C}=\mathrm{O}_{\text {ester }}\right), 1652$ $\left(\mathrm{C}=\mathrm{O}_{\text {pyridone }}\right)$ and $1609 \mathrm{~cm}^{-1}(\mathrm{C}=\mathrm{N})$. Also, its ${ }^{1} \mathrm{H} \mathrm{NMR}$ spectrum showed exchangeable signals at $\delta 5.60$ and $8.85 \mathrm{ppm}$ assigned to the $\mathrm{NH}_{2}$ and $\mathrm{NH}$ protons, respectively. The mass spectrum of compound 3 showed the molecular ion peak at $\mathrm{m} / \mathrm{z} 429$ which is coincident with the molecular weight (429.44) as supports the identity of the structure.

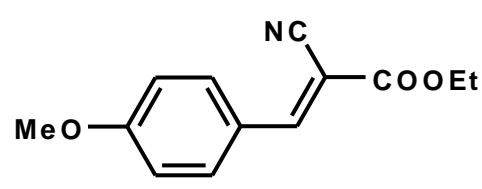

1

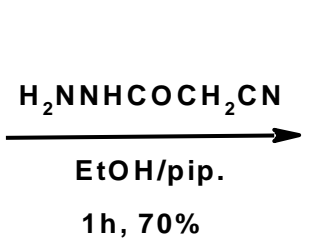

$1 \mathrm{~h}, 70 \%$<smiles>CCOC(=O)c1c(Br)c(C#N)c(=O)n(N)c1N</smiles>

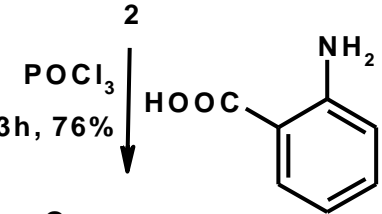<smiles>CCOC(=O)c1c(Br)c(C#N)c(=O)n2[nH]c(-c3ccccc3N)nc12</smiles>

3

Ar $=4-\mathrm{MeO}-\mathrm{C}_{6} \mathrm{H}_{4}$

Scheme 1. Synthesis of diaminopyridone 2 and triazolopyridone 3.

Compound $\mathbf{3}$ was used as a precursor for the synthesis of novel pyrido[1',2':2,3] $[1,2,4]$ triazolo[1,5-c] quinazoline derivatives via the reaction with some electrophilic reagents. Thus, heterocyclization of compound $\mathbf{3}$ with formic acid afforded ethyl 3-cyano-2-(4methoxyphenyl)-4-oxo-4,7-dihydro-pyrido[1',2':2,3][1,2,4]triazolo[1,5-c]quinazoline-1-

carboxylate (4) (Scheme 2). The ${ }^{1} \mathrm{H}$ NMR spectrum of compound 4 showed two characteristic singlet signals at $\delta 3.86$ and $8.63 \mathrm{ppm}$ assigned to the $\mathrm{OCH}_{3}$ and $\mathrm{H}-7$, respectively. The mass spectrum of compound 4 revealed the molecular ion peak at m/z 439 corresponding to the 
molecular formula $\mathrm{C}_{24} \mathrm{H}_{17} \mathrm{~N}_{5} \mathrm{O}_{4}$, which agree well with the molecular weight (439.43) and supports the identity of the structure.

Also, condensation of compound $\mathbf{3}$ with acetyl chloride and chloroacetyl chloride in boiling DMF containing few drops of triethylamine (TEA) gave pyrido[1',2':2,3][1,2,4]triazolo[1,5-c] quinazoline derivatives 5 and 6, respectively (Scheme 2). The ${ }^{1} \mathrm{H}$ NMR spectra of compounds 5 and 6 showed characteristic signals at $\delta 2.21$ and $2.73 \mathrm{ppm}$ assigned to the $\mathrm{CH}_{3}$ and $\mathrm{CH}_{2} \mathrm{Cl}$ protons, respectively. Also, the ${ }^{13} \mathrm{C}$ NMR spectrum of compound $\mathbf{5}$ showed characteristic signal attributed to $\mathrm{CH}_{3}$ pyrimidine at $\delta 20.6 \mathrm{ppm}$. Further, the mass spectra of compounds 5 and $\mathbf{6}$ revealed the molecular ion peaks at $\mathrm{m} / \mathrm{z} 453$ and 487 which agree well with the molecular weights for compounds $\mathbf{5}$ and $\mathbf{6}$, respectively.

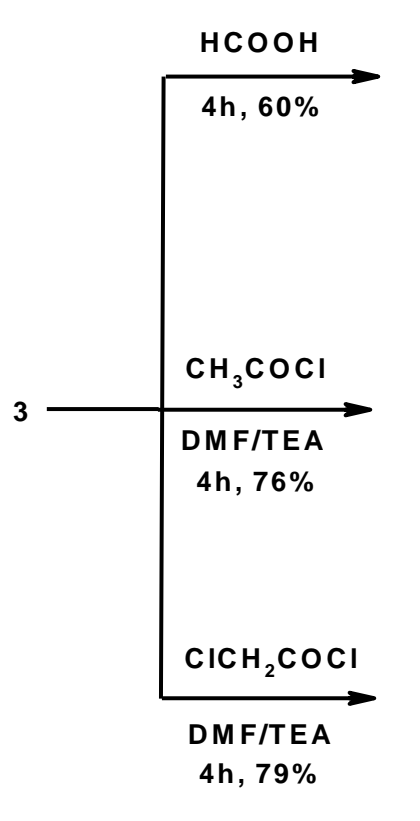

$\mathrm{Ar}=4-\mathrm{MeO}-\mathrm{C}_{6} \mathrm{H}_{4}$<smiles></smiles><smiles>CCOC(=O)c1c(Br)c(C#N)c(=O)n2c(C)nc3ccccc3c12</smiles>

5<smiles>CCOC(=O)c1c(Br)c(C#N)c(=O)n2c(CCl)nc3ccccc3c12</smiles>

6

Scheme 2. Synthesis of pyrido[1',2':2,3][1,2,4]triazolo[1,5-c]quinazolines 4-6.

In the same manner, cyclocondensation of the starting material $\mathbf{3}$ with ethyl chloroformate and $\mathrm{CS}_{2}$ in ethanolic potassium hydroxide solution afforded pyrido[1',2':2,3][1,2,4]triazolo[1,5-c] quinazolines $\mathbf{7}$ and $\mathbf{8}$, respectively (Scheme 3). The mass spectra of compounds $\mathbf{7}$ and $\mathbf{8}$ showed the molecular ion peaks at $\mathrm{m} / \mathrm{z} 455$ and 471 corresponding to the molecular formulas $\mathrm{C}_{24} \mathrm{H}_{17} \mathrm{~N}_{5} \mathrm{O}_{5}$ and $\mathrm{C}_{24} \mathrm{H}_{17} \mathrm{~N}_{5} \mathrm{O}_{4} \mathrm{~S}$, respectively. 


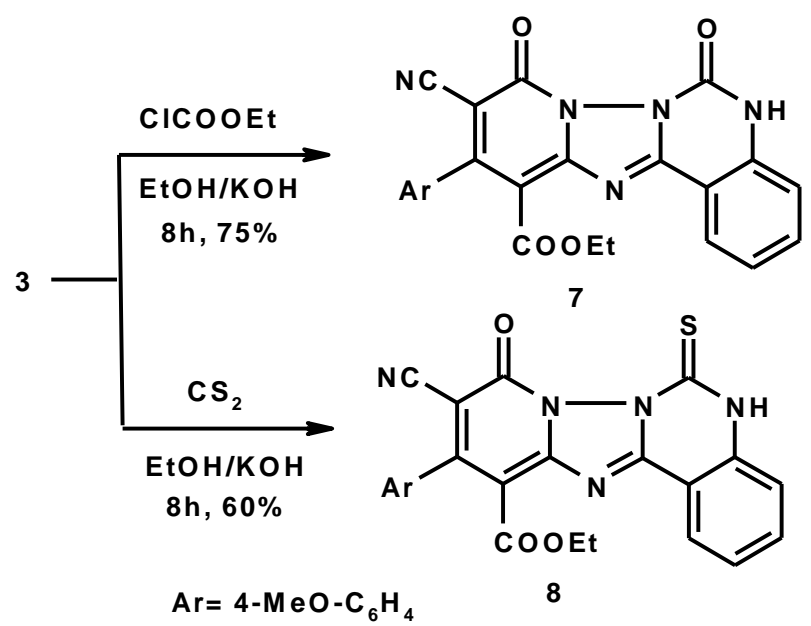

Scheme 3. Synthesis of pyrido[1',2':2,3][1,2,4]triazolo[1,5-c]quinazolines $\mathbf{7}$ and $\mathbf{8}$.

Next, the diaminopyridone derivative $\mathbf{2}$ was used as a starting material for the synthesis of a novel polyfused systems namely thiazolo[3',2':2,3][1,2,4]triazolo[1,5- $a]$ pyridines. Thus, condensation of compound 2 with $\mathrm{CS}_{2}$ in ethanolic $\mathrm{KOH}$ solution under reflux yielded ethyl 6cyano-7-(4-methoxyphenyl)-5-oxo-2-thioxo-1,2,3,5-tetrahydro[1,2,4]triazolo[1,5- $a$ ]pyridine-8carboxylate (9) (Scheme 4). ${ }^{18}$

Compound 9 was allowed to react with some bifunctional electrophiles to produce the corresponding thiazolo[ $\left[3^{\prime}, 2^{\prime}: 2,3\right][1,2,4]$ triazolo[ $[1,5-a]$ pyridines 10-13. Thus, heterocyclization of compound 9 with chloroacetic acid and oxalyl chloride in boiling DMF containing few drops of triethylamine produced the novel ethyl thiazolo[3',2':2,3][1,2,4]triazolo[1,5-a]pyridine-9carboxylate derivatives $\mathbf{1 0}$ and $\mathbf{1 1}$, respectively (Scheme 4). ${ }^{19}$

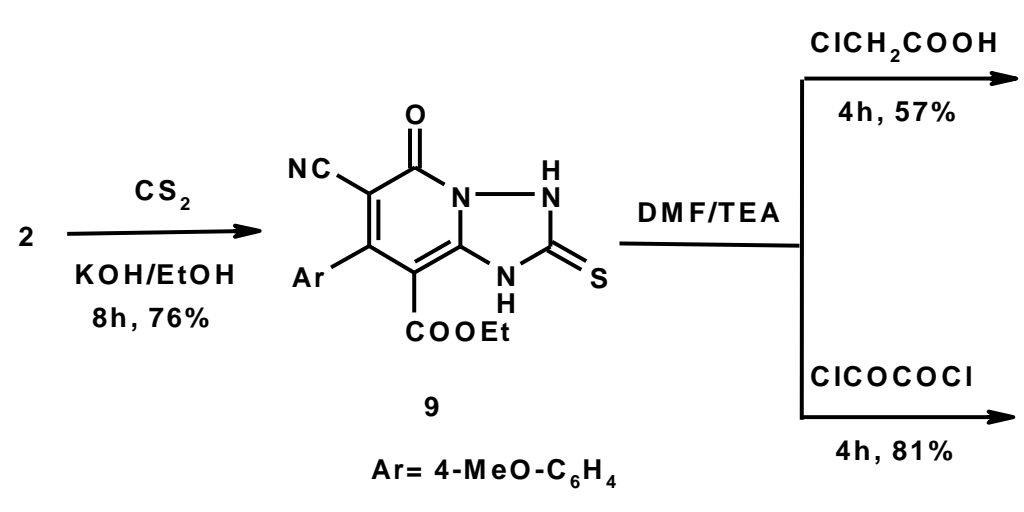<smiles>CCOC(=O)c1c(Br)c(C#N)c(=O)n2c3nc(=O)n(c1-3)SC2</smiles><smiles>CCOC(=O)c1c(Br)c(C#N)c(=O)n2c1nc1n2C(=O)C(=O)S1</smiles>

11

Scheme 4. Synthesis of thiazolo[3',2':2,3][1,2,4]triazolo[1,5- $a]$ pyridines 10 and $\mathbf{1 1}$. 
Treatment of compound 9 with chloroacetonitrile and bromomalononitrile in boiling DMF containing few drops of triethylamine produced ethyl 3-amino-7-cyano-8-(4-methoxyphenyl)-6oxo-6H-[1,3]thiazolo[3',2':2,3][1,2,4] triazolo[1,5-a]pyridine-9-carboxylate (12) and ethyl 3amino-2,7-dicyano-8-(4-methoxyphenyl)-6-oxo-6H-[1,3]thiazolo[3',2':2,3][1,2,4]triazolo[1,5-a] pyridine-9-carboxylate (13), respectively (Scheme 5). ${ }^{20}$ The ${ }^{1} \mathrm{H}$ NMR spectrum of compound 12 showed a characteristic singlet at $\delta 6.18 \mathrm{ppm}$ attributed to $\mathrm{H}-2$, in addition to an exchangeable signal at $\delta 8.37 \mathrm{ppm}$ assigned to the $\mathrm{NH}_{2}$ protons, while the ${ }^{1} \mathrm{H}$ NMR spectrum of compound $\mathbf{1 3}$ showed an exchangeable signal at $\delta 8.41 \mathrm{ppm}$ attributed to the $\mathrm{NH}_{2}$ protons.

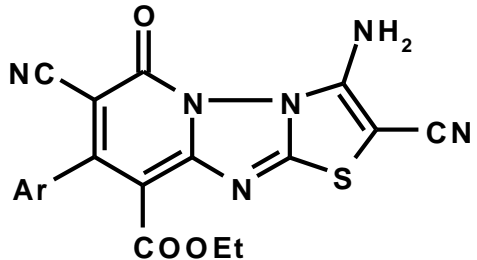

13

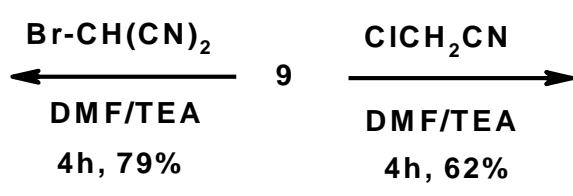

$4 \mathrm{~h}, 79 \%$

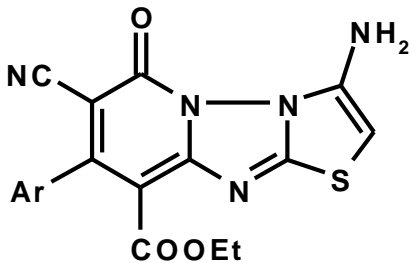

12

Scheme 5. Synthesis of thiazolo[3',2':2,3][1,2,4]triazolo[1,5- $a]$ pyridines 12 and 13.

\section{Antimicrobial activity}

The standardized disc agar diffusion method $^{21}$ was followed to determine the activity of the synthesized compounds against the sensitive organisms Staphylococcus aureus and Bacillus subtilis as Gram-positive bacteria, Salmonella typhimurium and Escherichia coli as Gramnegative bacteria and Candida albicans as fungus strain. The compounds were dissolved in DMSO which has no inhibition activity to get concentration of $100 \mu \mathrm{g} \mathrm{mL}{ }^{-1}$. The test was performed on medium potato dextrose agars (PDA) which contain infusion of $200 \mathrm{~g}$ potatoes, $6 \mathrm{~g}$ dextrose and $15 \mathrm{~g}$ agar. ${ }^{22}$ Uniform size filter paper disks (3 disks per compound) were impregnated by equal volume $(10 \mu \mathrm{L})$ from the specific concentration of dissolved tested compounds and carefully placed on inoculated agar surface. After incubation for $36 \mathrm{~h}$ at $27{ }^{\circ} \mathrm{C}$ in the case of bacteria and for $48 \mathrm{~h}$ at $24{ }^{\circ} \mathrm{C}$ in the case of fungi, inhibition of the organisms was measured and used to calculate mean of inhibition zones.

The diaminopyridone derivative $\mathbf{2}$ showed lower activity towards all types of microorganisms while the synthesized polyfused systems exhibited lower to mild antimicrobial activity. From the results depicted in Table 1, we can conclude that:

1. Pyrido[1',2':2,3][1,2,4]triazolo[1,5-c]quinazoline derivatives 4-8 showed lower activity towards most of the microorganisms except compounds $\mathbf{7}$ and $\mathbf{8}$ showed moderate activity toward Bacillus subtilis as Gram-positive bacteria, also compound $\mathbf{8}$ showed moderate activity towards Candida albicans as fungus strain.

2. Thiazolo[3',2':2,3][1,2,4]triazolo[1,5-a]pyridines derivatives 10-13 showed moderate activity toward Bacillus subtilis as Gram-positive bacteria, Salmonella typhimurium as Gram-negative 
bacteria and Candida albicans as fungus strain. Thus, the synthesized compounds may be considered promising for the development of new antimicrobial agents.

\section{Conclusions}

In the present work, a novel series of polynuclear pyrido[1',2':2,3][1,2,4]triazolo[1,5-c] quinazolines 4-8 and thiazolo[3',2':2,3][1,2,4]triazolo[1,5- $a$ ]pyridines $\mathbf{1 0 - 1 3}$ were efficiently synthesized via the key intermediate ethyl 1,2-diamino-5-cyano-4-(4-methoxyphenyl)-6-oxo-1,6dihydropyridine-3-carboxylate (2).

Table 1. The antimicrobial activity of the synthesized compounds $\mathbf{2 - 1 3}$

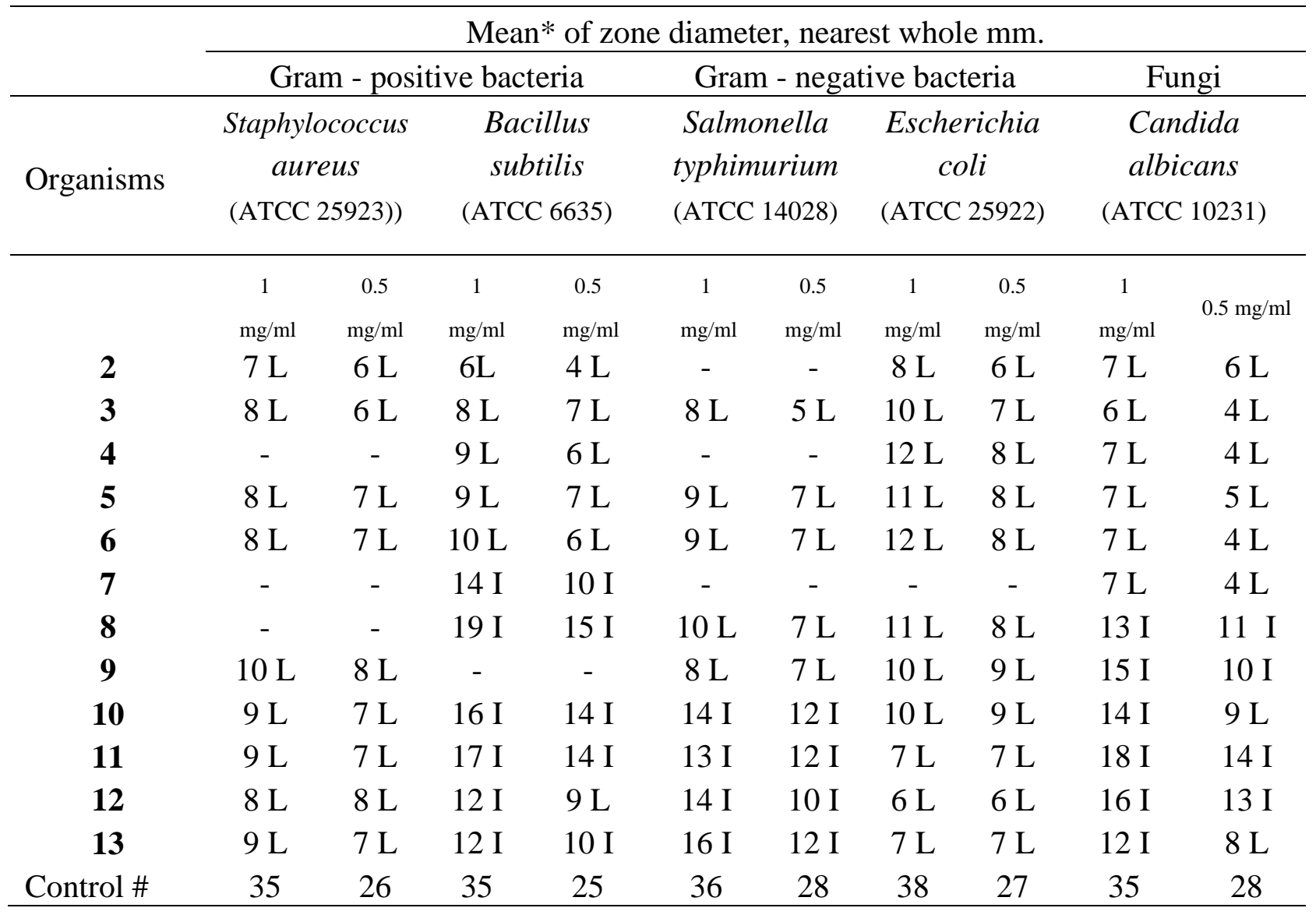

$*$ = Calculate from 3 values. - = No effect. L: Low activity $=$ Mean of zone diameter $\leq 1 / 3$ of mean zone diameter of control. I: Intermediate activity $=$ Mean of zone diameter $\leq 2 / 3$ of mean zone diameter of control. H: High activity = Mean of zone diameter $>2 / 3$ of mean zone diameter of control. \#: Chloramphenicol in the case of Gram-positive bacteria, Cephalothin in the case of Gram-negative bacteria and cycloheximide in the case of fungi. 


\section{Experimental Section}

General. Melting points were determined on a digital Stuart SMP3 apparatus. Infrared spectra were measured on FTIR Nicolet IS10 spectrophotometer $\left(\mathrm{cm}^{-1}\right)$, using $\mathrm{KBr}$ disks. ${ }^{1} \mathrm{H}$ NMR $(300 \mathrm{MHz})$ spectra were measured on Mercury-300BB, while ${ }^{13} \mathrm{C}$ NMR $(125 \mathrm{MHz})$ spectra were measured on Jeol-Eca500 MHz, using DMSO- $d_{6}$ as a solvent and TMS $(\delta)$ as the internal standard. Mass spectra were obtained using GC-2010 Shimadzu Gas chromatography mass spectrometry instrument $(70 \mathrm{eV})$. Elemental microanalyses were performed on a Perkin-Elmer CHN-2400 analyzer.

\section{Ethyl 1,2-diamino-5-cyano-4-(4-methoxyphenyl)-6-oxo-1,6-dihydropyridine-3-carboxylate} (2). A mixture of ethyl 3-cyano-2-(4-methoxyphenyl)prop-2-enoate (1) $(2.31 \mathrm{~g}, 10 \mathrm{mmol})$ and cyanoacetohydrazide, $(0.99 \mathrm{~g}, 10 \mathrm{mmol})$, in absolute ethanol $(40 \mathrm{~mL})$ containing two drops of piperidine, was heated under reflux for $1 \mathrm{~h}$. The yellow crystals obtained during heating were filtered off and crystallized from DMF/EtOH to give compound 2 as white crystals, yield $2.33 \mathrm{~g}$ (70\%), mp 215-216 ${ }^{\circ} \mathrm{C}$. IR $\left(\mathrm{KBr}, \mathrm{cm}^{-1}\right): 3216,3089,3079\left(2 \mathrm{NH}_{2}\right), 3012\left(\mathrm{CH}_{\text {arom. }}\right), 2965,2947$, 2919, $2836\left(\mathrm{CH}_{\text {aliph. }}\right), 2255(\mathrm{C} \equiv \mathrm{N}), 1692\left(\mathrm{C}=\mathrm{O}_{\text {ester }}\right), 1672\left(\mathrm{C}=\mathrm{O}_{\text {pyridone }}\right), 1607(\mathrm{C}=\mathrm{C}) .{ }^{1} \mathrm{H}$ NMR $\left(\mathrm{DMSO}-d_{6}, \delta\right): 1.22\left(\mathrm{t}, 3 \mathrm{H}, \mathrm{CH}_{3}\right), 3.77\left(\mathrm{~s}, 3 \mathrm{H}, \mathrm{OCH}_{3}\right), 4.15\left(\mathrm{q}, 2 \mathrm{H}, \mathrm{CH}_{2}\right), 5.62\left(\mathrm{bs}, 2 \mathrm{H}, \mathrm{N}-\mathrm{NH}_{2}\right.$ exchangeable with $\left.\mathrm{D}_{2} \mathrm{O}\right), 6.97(\mathrm{~d}, 2 \mathrm{H}, \operatorname{Ar}-\mathrm{H}), 7.62(\mathrm{~d}, 2 \mathrm{H}, \mathrm{Ar}-\mathrm{H}), 8.01$ (bs, $1 \mathrm{H}, \mathrm{C}-\mathrm{NH}_{2}$ exchangeable with $\mathrm{D}_{2} \mathrm{O}$ ), 8.81 (bs, $1 \mathrm{H}, \mathrm{C}-\mathrm{NH}_{2}$ exchangeable with $\mathrm{D}_{2} \mathrm{O}$ ). ${ }^{13} \mathrm{C}$ NMR (DMSO- $d_{6}$, $\delta): 12.9\left(\mathrm{CH}_{3}\right), 55.8\left(\mathrm{OCH}_{3}\right), 62.0\left(\mathrm{CH}_{2}\right), 87.2(\mathrm{C}-5), 114.8,114.9,116.6,126.9,129.4,148.2$, 159.1, 161.4, 161.6, 165.0. m/z (I \%): 328 (45), 312 (43), 298 (68), 284 (47), 212 (68), 196 (66), 157 (57), 130 (54), 107 (46), 77 (91), 69 (100). Analysis Calcd for $\mathrm{C}_{16} \mathrm{H}_{16} \mathrm{~N}_{4} \mathrm{O}_{4}$ (328.33); C, $58.53 ; \mathrm{H}, 4.91 ; \mathrm{N}, 17.06 \%$. Found: C, 58.36; H, 4.72; N, $16.84 \%$.

Ethyl 2-[(2-aminophenyl)]-6-cyano-7-[(4-methoxyphenyl)]-5-oxo-3,5-dihydro-[1,2,4] triazolo[1,5-a]pyridine-8-carboxylate (3). A mixture of compound $2(0.66 \mathrm{~g}, 2 \mathrm{mmol})$ and anthranilic acid $(0.27 \mathrm{~g}, 2 \mathrm{mmol})$ in $\mathrm{POCl}_{3}(10 \mathrm{~mL})$ was heated under reflux on a water bath for $3 \mathrm{~h}$. After cooling, the reaction mixture was poured onto ice/water. The precipitated solid was filtered off, washed with water, air dried and crystallized from $\mathrm{DMF} / \mathrm{MeOH}$ to give compound 3 as yellow crystals, yield $0.65 \mathrm{~g}(76 \%)$, mp $186-187^{\circ} \mathrm{C}$. IR $\left(\mathrm{KBr}, \mathrm{cm}^{-1}\right): 3417,3341,3241\left(\mathrm{NH}_{2}\right.$, $\mathrm{NH}), 3005\left(\mathrm{CH}_{\text {arom. }}\right), 2950,2932,2837 \quad\left(\mathrm{CH}_{\text {aliph. }}\right), 2189(\mathrm{C} \equiv \mathrm{N}), 1678\left(\mathrm{C}=\mathrm{O}_{\text {ester }}\right), 1652$ $\left(\mathrm{C}=\mathrm{O}_{\text {pyridone }}\right), 1609(\mathrm{C}=\mathrm{N}), 1585(\mathrm{C}=\mathrm{C}) .{ }^{1} \mathrm{H}$ NMR $\left(\mathrm{DMSO}-d_{6}\right): 1.23\left(\mathrm{t}, 3 \mathrm{H}, \mathrm{CH}_{3}\right), 3.72(\mathrm{q}, 2 \mathrm{H}$, $\mathrm{CH}_{2}$ ), 3.85 (s, $\left.3 \mathrm{H}, \mathrm{OCH}_{3}\right), 5.60$ (bs, $2 \mathrm{H}, \mathrm{NH}_{2}$ exchangeable with $\mathrm{D}_{2} \mathrm{O}$ ), 6.81-6.87 (m, $\left.2 \mathrm{H}, \mathrm{Ar}-\mathrm{H}\right)$, 7.02-7.36 (m, 4H, Ar-H), 7.50-7.64 (m, 2H, Ar-H), 8.85 (bs, $1 \mathrm{H}, \mathrm{NH}$ exchangeable with $\left.\mathrm{D}_{2} \mathrm{O}\right)$. m/z (I \%): 429 (11), 415 (22), 403 (28), 385 (14), 372 (69), 356 (32), 339 (85), 310 (98), 252 (18), 119 (24), 108 (23), 78 (7), 64 (100). Analysis Calcd for $\mathrm{C}_{23} \mathrm{H}_{19} \mathrm{~N}_{5} \mathrm{O}_{4}$ (429.44); C, 64.33; H, 4.46; N, 16.31\%. Found: C, 64.08; H, 4.34; N, 16.17\%.

Ethyl 3-cyano-2-(4-methoxyphenyl)-4-oxo-4,7-dihydro-pyrido[1',2':2,3][1,2,4]triazolo[1,5c]quinazoline-1-carboxylate (4). A mixture of compound $3(0.86 \mathrm{~g}, 2 \mathrm{mmol})$ and formic acid $(10 \mathrm{~mL})$ was heated under reflux for $4 \mathrm{~h}$. After cooling, the reaction mixture was poured onto 
ice/water. The precipitated solid was filtered off, washed with water, air dried and crystallized from EtOH to give compound 4 as yellow crystals, yield $0.53 \mathrm{~g}(60 \%), \mathrm{mp} 201-202{ }^{\circ} \mathrm{C}$. IR $(\mathrm{KBr}$, $\left.\mathrm{cm}^{-1}\right)$ : $3020\left(\mathrm{CH}_{\text {arom. }}\right), 2935,2922,2851\left(\mathrm{CH}_{\text {aliph. }}\right), 2219(\mathrm{C} \equiv \mathrm{N}), 1654\left(\mathrm{C}=\mathrm{O}_{\text {ester }}\right.$ and $\left.\mathrm{C}=\mathrm{O}_{\text {pyridone }}\right)$, $1607(\mathrm{C}=\mathrm{N}), 1586(\mathrm{C}=\mathrm{C}) .{ }^{1} \mathrm{H}$ NMR (DMSO-d $)$ : $1.17\left(\mathrm{t}, 3 \mathrm{H}, \mathrm{CH}_{3}\right), 3.71\left(\mathrm{q}, 2 \mathrm{H}, \mathrm{CH}_{2}\right), 3.86(\mathrm{~s}$, $\left.3 \mathrm{H}, \mathrm{OCH}_{3}\right), 6.83(\mathrm{~d}, 2 \mathrm{H}, \mathrm{Ar}-\mathrm{H}, J 8.7 \mathrm{~Hz}), 7.05(\mathrm{~d}, 2 \mathrm{H}, \mathrm{Ar}-\mathrm{H}, J 8.7 \mathrm{~Hz}), 7.51(\mathrm{t}, 1 \mathrm{H}, \mathrm{Ar}-\mathrm{H}, J 8.7$ $\mathrm{Hz}), 7.61(\mathrm{~d}, 1 \mathrm{H}, \mathrm{Ar}-\mathrm{H}, J 8.7 \mathrm{~Hz}), 7.67-7.88(\mathrm{~m}, 2 \mathrm{H}, \mathrm{Ar}-\mathrm{H}), 8.63(\mathrm{~s}, 1 \mathrm{H}, \mathrm{H}-7$ as $\mathrm{CH}=\mathrm{N}) . m / z(I$ \%): 439 (2), 411 (2), 366 (3), 336 (6), 306 (4), 280 (3), 262 (2), 107 (13), 93 (11), 77 (5), 57 (100). Analysis Calcd for $\mathrm{C}_{24} \mathrm{H}_{17} \mathrm{~N}_{5} \mathrm{O}_{4}$ (439.43); C, 65.60; H, 3.90; N, 15.94\%. Found: C, 65.42; H, 3.82; N, $15.71 \%$.

Ethyl 3-cyano-2-(4-methoxyphenyl)-7-methyl-4-oxo-4H-pyrido[1',2':2,3][1,2,4]triazolo[1,5c]quinazoline-1-carboxylate (5). A mixture of compound 3 (0.86 g, $2 \mathrm{mmol})$ and acetyl chloride $(0.14 \mathrm{~mL}, 2 \mathrm{mmol})$, in DMF containing few drops of triethylamine, was heated under reflux for $4 \mathrm{~h}$. After cooling, the reaction mixture was poured onto ice/water. The precipitated solid was filtered off, washed with water, air dried and crystallized from DMF to give compound

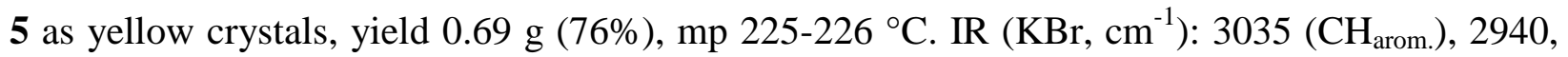
2924, $2852\left(\mathrm{CH}_{\text {aliph. }}\right), 2216(\mathrm{C} \equiv \mathrm{N}), 1685\left(\mathrm{C}=\mathrm{O}_{\text {ester }}\right.$ and $\left.\mathrm{C}=\mathrm{O}_{\text {pyridone }}\right), 1607(\mathrm{C}=\mathrm{N}), 1597(\mathrm{C}=\mathrm{C}) .{ }^{1} \mathrm{H}$ NMR (DMSO-d $)$ : $1.16\left(\mathrm{t}, 3 \mathrm{H}, \mathrm{CH}_{3}\right), 2.21\left(\mathrm{~s}, 3 \mathrm{H}, \mathrm{CH}_{3}\right), 3.71$ (q, 2H, $\left.\mathrm{CH}_{2}\right), 3.86\left(\mathrm{~s}, 3 \mathrm{H}, \mathrm{OCH}_{3}\right)$, $6.82(\mathrm{~d}, 2 \mathrm{H}, \mathrm{Ar}-\mathrm{H}, J 8.7 \mathrm{~Hz}), 7.09$ (d, 2H, Ar-H, J 8.7 Hz), 7.43-7.81 (m, 4H, Ar-H). ${ }^{13} \mathrm{C}$ NMR (DMSO-d $\left.d_{6} \delta\right): 12.7\left(\mathrm{CH}_{3}\right), 20.6\left(\mathrm{CH}_{3}\right.$ pyrimidine $), 56.6\left(\mathrm{OCH}_{3}\right), 61.8\left(\mathrm{CH}_{2}\right), 110.4,114.9,116.3$, $116.5,118.1,128.0,128.6,130.5,131.8,131.9,134.5,147.1,147.2,151.6,158.2,161.3,161.6$, 166.9, 167.1. m/z (I \%): 453 (14), 438 (7), 425 (21), 399 (17), 384 (34), 314 (18), 289 (26), 247 (43), 155 (54), 98 (49), 81 (73), 57 (100). Analysis Calcd for $\mathrm{C}_{25} \mathrm{H}_{19} \mathrm{~N}_{5} \mathrm{O}_{4}$ (453.46); C, 66.22; $\mathrm{H}$, 4.22; N, 15.44\%. Found: C, 66.03; H, 3.94; N, 15.12\%.

Ethyl 7-chloromethyl-3-cyano-2-(4-methoxyphenyl)-4-oxo-4H-pyrido[1',2':2,3][1,2,4] triazolo[1,5-c]quinazoline-1-carboxylate (6). A mixture of compound 3 (0.86 g, $2 \mathrm{mmol})$ and chloroacetyl chloride $(0.16 \mathrm{~mL}, 2 \mathrm{mmol})$, in DMF containing few drops of triethylamine, was heated under reflux for $4 \mathrm{~h}$. After cooling, the reaction mixture was poured onto ice/water. The precipitated solid was filtered off, washed with water, air dried and crystallized from $\mathrm{DMF} / \mathrm{H}_{2} \mathrm{O}$ to give compound 6 as yellow crystals, yield $0.77 \mathrm{~g}(79 \%), \mathrm{mp} 126-127^{\circ} \mathrm{C}$. IR $\left(\mathrm{KBr}, \mathrm{cm}^{-1}\right): 3003$ $\left(\mathrm{CH}_{\text {arom. }}\right), 2931,2831\left(\mathrm{CH}_{\text {aliph. }}\right), 2222(\mathrm{C} \equiv \mathrm{N}), 1683\left(\mathrm{C}=\mathrm{O}_{\text {ester }}\right), 1651\left(\mathrm{C}=\mathrm{O}_{\text {pyridone }}\right), 1608(\mathrm{C}=\mathrm{N})$, $1576(\mathrm{C}=\mathrm{C}) .{ }^{1} \mathrm{H}$ NMR (DMSO-d $): 1.22\left(\mathrm{t}, 3 \mathrm{H}, \mathrm{CH}_{3}\right), 2.73\left(\mathrm{~s}, 2 \mathrm{H}, \mathrm{CH}_{2} \mathrm{Cl}\right.$ ), 3.70 (q, 2H, $\mathrm{CH}_{2}$ ), $3.84\left(\mathrm{~s}, 3 \mathrm{H}, \mathrm{OCH}_{3}\right), 6.82-7.85(\mathrm{~m}, 8 \mathrm{H}, \mathrm{Ar}-\mathrm{H}) . \mathrm{m} / \mathrm{z}$ (I \%): $489(\mathrm{M}+2 ; 4), 487\left(\mathrm{M}^{+}, 12\right), 472(5)$, 458 (34), 363 (9), 252 (33), 227 (24), 149 (39), 135 (18), 92 (26), 57 (100). Analysis Calcd for $\mathrm{C}_{25} \mathrm{H}_{18} \mathrm{ClN}_{5} \mathrm{O}_{4}$ (487.91); C, 61.54; H, 3.72; N, 14.35\%. Found: C, 61.19; H, 3.54; N, 14.17\%.

Ethyl 3-cyano-2-(4-methoxyphenyl)-4,7-dioxo-4,7-dihydro-8H-pyrido[1',2':2,3][1,2,4] triazolo[1,5-c]quinazoline-1-carboxylate (7). A mixture of compound $3(0.86 \mathrm{~g}, 2 \mathrm{mmol})$ and ethyl chloroformate $(0.22 \mathrm{~g}, 0.2 \mathrm{~mL}, 2 \mathrm{mmol})$ in ethanolic potassium hydroxide $(2 \%, 30 \mathrm{~mL})$ was heated under reflux for $8 \mathrm{~h}$. After cooling, the reaction mixture was poured onto ice/water and neutralized with conc. $\mathrm{HCl}$. The precipitated solid was filtered off, washed with water, air dried and crystallized from $\mathrm{DMF} / \mathrm{EtOH}$ to give compound 7 as yellow crystals, yield $0.68 \mathrm{~g}$ 
(75\%), mp 300-301 ${ }^{\circ} \mathrm{C}$. IR ( $\left.\mathrm{KBr}, \mathrm{cm}^{-1}\right): 3423(\mathrm{NH}), 2933,2921,2850\left(\mathrm{CH}_{\text {aliph. }}\right), 2213(\mathrm{C} \equiv \mathrm{N})$, $1680\left(\mathrm{C}=\mathrm{O}_{\text {ester }}\right), 1651\left(\mathrm{C}=\mathrm{O}_{\text {pyridone }}\right.$ and $\left.\mathrm{C}=\mathrm{O}_{\text {pyrimidinone }}\right), 1606(\mathrm{C}=\mathrm{N}$ and $\mathrm{C}=\mathrm{C}) .{ }^{1} \mathrm{H}$ NMR (DMSO$\left.d_{6}\right): 1.15\left(\mathrm{t}, 3 \mathrm{H}, \mathrm{CH}_{3}\right), 3.69$ (q, 2H, $\left.\mathrm{CH}_{2}\right), 3.84\left(\mathrm{~s}, 3 \mathrm{H}, \mathrm{OCH}_{3}\right), 6.84-7.65$ (m, 8H, Ar-H). $m / z(I$ \%): 455 (3), 454 (8), 399 (2), 358 (8), 284 (3), 271 (13), 256 (16), 238 (18), 135 (88), 120 (87), 77 (19), 57 (100). Analysis Calcd for $\mathrm{C}_{24} \mathrm{H}_{17} \mathrm{~N}_{5} \mathrm{O}_{5}$ (455.43); C, 63.30; H, 3.76; N, 15.38\%. Found: C, 63.21; H, 3.58; N, 15.14\%.

Ethyl 3-cyano-2-(4-methoxyphenyl)-4-oxo-7-thioxo-4,7-dihydro-8H-pyrido[1',2':2,3] $[1,2,4]$ triazolo[1,5-c]quinazoline-1-carboxylate (8). A mixture of compound $3(0.86 \mathrm{~g}, 2$ $\mathrm{mmol})$ and carbon disulfide $(0.12 \mathrm{~mL}, 2 \mathrm{mmol})$ in ethanolic potassium hydroxide solution $(2 \%$, $30 \mathrm{~mL}$ ) was heated under reflux for $8 \mathrm{~h}$. After cooling, the reaction mixture was poured onto ice/water and neutralized with conc. $\mathrm{HCl}$. The precipitated solid was filtered off, washed with water, air dried and crystallized from $\mathrm{DMF} / \mathrm{EtOH}$ to give compound $\mathbf{8}$ as yellow crystals, yield $0.56 \mathrm{~g}(60 \%), \mathrm{mp} 231-232{ }^{\circ} \mathrm{C}$. IR $\left(\mathrm{KBr}, \mathrm{cm}^{-1}\right): 3420(\mathrm{NH}), 2938,2925,2845\left(\mathrm{CH}_{\text {aliph. }}\right), 2216$ $(\mathrm{C} \equiv \mathrm{N}), 1686\left(\mathrm{C}=\mathrm{O}_{\text {ester }}\right), 1654\left(\mathrm{C}=\mathrm{O}_{\text {pyridone }}\right), 1607(\mathrm{C}=\mathrm{N}), 1576(\mathrm{C}=\mathrm{C}), 1251(\mathrm{C}=\mathrm{S}) .{ }^{1} \mathrm{H}$ NMR (DMSO-d $): 1.24$ (t, 3H, $\mathrm{CH}_{3}$ ), 3.72 (q, 2H, $\mathrm{CH}_{2}$ ), 3.86 (s, 3H, OCH 3 ), 6.85 (d, 2H, Ar-H, J 9.0 $\mathrm{Hz}$ ), 7.12 (d, 2H, Ar-H, J 9.0 Hz), 7.40-7.94 (m, 4H, Ar-H), 8.20 (bs, 1H, NH exchangeable with $\left.\mathrm{D}_{2} \mathrm{O}\right) . m / z$ (I \%): 471 (76), 442 (9), 363 (33), 256 (35), 224 (16), 80 (39), 64 (100). Analysis Calcd for $\mathrm{C}_{24} \mathrm{H}_{17} \mathrm{~N}_{5} \mathrm{O}_{4} \mathrm{~S}$ (471.50); C, 61.14; H, 3.63; N, 14.85; S, 6.80\%. Found: C, 61.11; H, $3.58 ; \mathrm{N}, 14.57 ; \mathrm{S}, 6.61 \%$.

Ethyl 6-cyano-7-(4-methoxyphenyl)-5-oxo-2-thioxo-1,2,3,5-tetrahydro[1,2,4]triazolo[1,5-a] pyridine-8-carboxylate (9). A mixture of compound $3(0.67 \mathrm{~g}, 2 \mathrm{mmol})$ and carbon disulfide $(0.12 \mathrm{~mL}, 2 \mathrm{mmol})$ in ethanolic potassium hydroxide solution $(2 \%, 30 \mathrm{~mL})$ was heated under reflux for $8 \mathrm{~h}$. After cooling, the reaction mixture was poured onto ice/water and neutralized with conc. $\mathrm{HCl}$. The precipitated solid was filtered off, washed with water, air dried and crystallized from $\mathrm{DMF} / \mathrm{EtOH}$ to give compound 9 as yellow crystals, yield $0.56 \mathrm{~g}(76 \%), \mathrm{mp} 174-175^{\circ} \mathrm{C}$. IR $\left(\mathrm{KBr}, \mathrm{cm}^{-1}\right): 3279(2 \mathrm{NH}), 3110\left(\mathrm{CH}_{\text {arom. }}\right), 2910,2854\left(\mathrm{CH}_{\text {aliph. }}\right), 2205(\mathrm{C} \equiv \mathrm{N}), 1683\left(\mathrm{C}=\mathrm{O}_{\text {ester }}\right.$ and $\left.\mathrm{C}=\mathrm{O}_{\text {pyridone }}\right), 1588(\mathrm{C}=\mathrm{C}),{ }^{1} \mathrm{H}$ NMR (DMSO-d $): 1.17\left(\mathrm{t}, 3 \mathrm{H}, \mathrm{CH}_{3}, J 7.2 \mathrm{~Hz}\right), 3.04\left(\mathrm{q}, 2 \mathrm{H}, \mathrm{CH}_{2}, J\right.$ $7.2 \mathrm{~Hz}$ ), 3.82 (s, 3H, $\left.\mathrm{OCH}_{3}\right), 7.04$ (d, 2H, Ar-H, J 9.0 Hz), 7.80 (d, 2H, Ar-H, J 8.7 Hz), 8.63 (bs, $2 \mathrm{H}, 2 \mathrm{NH}$ exchangeable with $\left.\mathrm{D}_{2} \mathrm{O}\right)$. Analysis Calcd for $\mathrm{C}_{17} \mathrm{H}_{14} \mathrm{~N}_{4} \mathrm{O}_{4} \mathrm{~S}$ (370.39); C, 55.13; H, 3.81; N, 15.13; S, 8.66\%. Found: C, 55.21; H, 3.65; N, 14.92; S, 8.38\%.

Ethyl 7-cyano-8-(4-methoxyphenyl)-3,6-dioxo-2,3-dihydro-6H-[1,3]thiazolo[3',2':2,3] $[1,2,4]$ triazolo[1,5-a]pyridine-9-carboxylate (10). A mixture of compound 9 (0.74 g, 2 mmol) and chloroacetic acid $(0.17 \mathrm{~g}, 2 \mathrm{mmol})$, in DMF $(10 \mathrm{~mL})$ containing few drops of triethylamine, was heated under reflux for $4 \mathrm{~h}$. After cooling, the reaction mixture was poured onto ice/water. The precipitated solid was filtered off, washed with water, air dried and crystallized from methanol to give compound $\mathbf{1 0}$ as yellow crystals, yield $0.47 \mathrm{~g}(57 \%), \mathrm{mp} 298-299^{\circ} \mathrm{C}$. IR $(\mathrm{KBr}$, $\left.\mathrm{cm}^{-1}\right)$ : $3033\left(\mathrm{CH}_{\text {arom. }}\right), 2924,2890\left(\mathrm{CH}_{\text {aliph. }}\right), 2224(\mathrm{C} \equiv \mathrm{N}), 1706\left(\mathrm{C}=\mathrm{O}_{\text {ester }}\right), 1657\left(\mathrm{C}=\mathrm{O}_{\text {pyridone }}\right)$, $1603(\mathrm{C}=\mathrm{N}$ and $\mathrm{C}=\mathrm{C}) .{ }^{1} \mathrm{H} \mathrm{NMR}\left(\mathrm{DMSO}-d_{6}\right): 1.16\left(\mathrm{t}, 3 \mathrm{H}, \mathrm{CH}_{3}\right), 3.09\left(\mathrm{q}, 2 \mathrm{H}, \mathrm{CH}_{2}\right), 3.65(\mathrm{~s}, 2 \mathrm{H}$, $\left.\mathrm{CH}_{2} \mathrm{C}=\mathrm{O}\right), 3.87\left(\mathrm{~s}, 3 \mathrm{H}, \mathrm{OCH}_{3}\right), 7.56(\mathrm{~d}, 2 \mathrm{H}, \mathrm{Ar}-\mathrm{H}), 7.84(\mathrm{~d}, 2 \mathrm{H}, \mathrm{Ar}-\mathrm{H}) .{ }^{13} \mathrm{C}$ NMR (DMSO- $\left.d_{6}, \delta\right)$ : $13.1\left(\mathrm{CH}_{3}\right), 36.8\left(\mathrm{CH}_{2}\right.$ thiazole $), 55.5\left(\mathrm{OCH}_{3}\right), 62.4\left(\mathrm{CH}_{2}\right), 103.2,114.6,114.9,116.8,128.9,130.8$, 
148.0, 149.2, 158.0, 161.4, 162.6, 165.5, 167.2. Analysis Calcd for $\mathrm{C}_{19} \mathrm{H}_{14} \mathrm{~N}_{4} \mathrm{O}_{5} \mathrm{~S}$ (410.41); C, 55.61; H, 3.44; N, 13.65; S, 7.81\%. Found: C, 55.48; H, 3.30; N, 13.52; S, 7.72\%.

Ethyl 7-cyano-8-(4-methoxyphenyl)-2,3,6-trioxo-2,3-dihydro-6H-[1,3]thiazolo[3',2':2,3] $[1,2,4]$ triazolo[1,5-a]pyridine-9-carboxylate (11). A mixture of compound 9 (0.74 g, 2 mmol) and oxalyl chloride $(0.18 \mathrm{~mL}, 2 \mathrm{mmol})$, in DMF $(10 \mathrm{~mL})$ containing few drops of triethylamine, was heated under reflux for $4 \mathrm{~h}$. After cooling, the reaction mixture was poured onto ice/water. The precipitated solid was filtered off, washed with water, air dried and crystallized from DMF to give compound 11 as yellow crystals, yield $0.69 \mathrm{~g}(81 \%), \mathrm{mp} 274-275^{\circ} \mathrm{C}$. IR $\left(\mathrm{KBr}, \mathrm{cm}^{-1}\right)$ : $3080\left(\mathrm{CH}_{\text {arom. }}\right), 2924,2848\left(\mathrm{CH}_{\text {aliph. }}\right), 2223(\mathrm{C} \equiv \mathrm{N}), 1739,1660(4 \mathrm{C}=\mathrm{O}), 1580(\mathrm{C}=\mathrm{N})$ and 1543 $(\mathrm{C}=\mathrm{C}) .{ }^{1} \mathrm{H}$ NMR (DMSO-d $)$ ) 1.14 (t, 3H, $\mathrm{CH}_{3}$ ), 3.03 (q, 2H, $\mathrm{CH}_{2}$ ), 3.87 (s, 3H, $\mathrm{OCH}_{3}$ ), 7.49 (d, $2 \mathrm{H}, \mathrm{Ar}-\mathrm{H}), 7.75$ (d, 2H, Ar-H). Analysis Calcd for $\mathrm{C}_{19} \mathrm{H}_{12} \mathrm{~N}_{4} \mathrm{O}_{6} \mathrm{~S}$ (424.39); C, 53.77; H, 2.85; N, 13.20; S, 7.56\%. Found: C, 53.41; H, 2.56; N, 12.84; S, 7.32\%.

Ethyl 3-amino-7-cyano-8-(4-methoxyphenyl)-6-oxo-6H-[1,3]thiazolo[3', $\left.2^{\prime}: 2,3\right][1,2,4]$ triazolo[1,5-a]pyridine-9-carboxylate (12). A mixture of compound 9 (0.74 g, 2 mmol) and chloroacetonitrile $(0.12 \mathrm{~mL}, 2 \mathrm{mmol})$, in DMF $(10 \mathrm{~mL})$ containing few drops of triethylamine, was heated under reflux for $4 \mathrm{~h}$. After cooling, the reaction mixture was poured onto ice/water. The precipitated solid was filtered off, washed with water, air dried and crystallized from methanol to give compound 12 as yellow crystals, yield $0.51 \mathrm{~g}(62 \%), \mathrm{mp} 257-258^{\circ} \mathrm{C}$. IR $(\mathrm{KBr}$, $\left.\mathrm{cm}^{-1}\right)$ : 3436, $3280\left(\mathrm{NH}_{2}\right), 3016\left(\mathrm{CH}_{\text {arom. }}\right), 2960,2833\left(\mathrm{CH}_{\text {aliph. }}\right), 2224(\mathrm{C} \equiv \mathrm{N}), 1718\left(\mathrm{C}=\mathrm{O}_{\text {ester }}\right)$, $1670\left(\mathrm{C}=\mathrm{O}_{\text {pyridone}}\right), 1596(\mathrm{C}=\mathrm{N}), 1554(\mathrm{C}=\mathrm{C}) .{ }^{1} \mathrm{H}$ NMR (DMSO-d $)$ : $1.27\left(\mathrm{t}, 3 \mathrm{H}, \mathrm{CH}_{3}\right), 3.74(\mathrm{q}$, $\left.2 \mathrm{H}, \mathrm{CH}_{2}\right), 3.89$ (s, 3H, OCH 3$), 6.18(\mathrm{~s}, 1 \mathrm{H}, \mathrm{H}-2), 7.09$ (d, 2H, Ar-H, J 7.5 Hz), 7.46 (d, 2H, Ar$\mathrm{H}, J 7.5 \mathrm{~Hz}$ ), 8.37 (bs, $2 \mathrm{H}, \mathrm{NH}_{2}$ exchangeable with $\mathrm{D}_{2} \mathrm{O}$ ). Analysis Calcd for $\mathrm{C}_{19} \mathrm{H}_{15} \mathrm{~N}_{5} \mathrm{O}_{4} \mathrm{~S}$ (409.43); C, 55.74; H, 3.69; N, 17.11; S, 7.83\%. Found: C, 55.52; H, 3.72; N, 17.14; S, 7.59\%.

Ethyl 3-amino-2,7-dicyano-8-(4-methoxyphenyl)-6-oxo-6H-[1,3]thiazolo[3',2':2,3][1,2,4] triazolo[1,5-a]pyridine-9-carboxylate (13). A mixture of compound 9 (0.74 g, $2 \mathrm{mmol})$ and bromomalononitrile $(2 \mathrm{mmol})$, in DMF $(10 \mathrm{~mL})$ containing few drops of triethylamine, was heated under reflux for $4 \mathrm{~h}$. After cooling, the reaction mixture was poured onto ice/water. The precipitated solid was filtered off, washed with water, air dried and crystallized from ethanol to

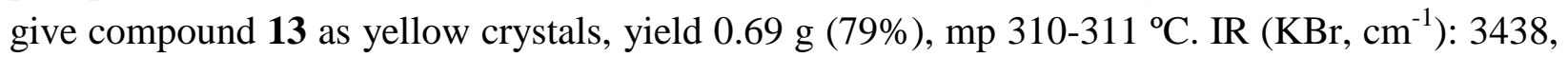
$3332\left(\mathrm{NH}_{2}\right), 3104\left(\mathrm{CH}_{\text {arom. }}\right), 2925,2850\left(\mathrm{CH}_{\text {aliph. }}\right), 2219(2 \mathrm{C} \equiv \mathrm{N}), 1695\left(\mathrm{C}=\mathrm{O}_{\text {ester }}\right.$ and $\left.\mathrm{C}=\mathrm{O}_{\text {pyridone }}\right)$, $1619(\mathrm{C}=\mathrm{N}), 1548(\mathrm{C}=\mathrm{C}) .{ }^{1} \mathrm{H}$ NMR (DMSO-d $): 1.26\left(\mathrm{t}, 3 \mathrm{H}, \mathrm{CH}_{3}\right), 3.03$ (q, 2H, $\left.\mathrm{CH}_{2}\right), 3.97$ (s, $\left.3 \mathrm{H}, \mathrm{OCH}_{3}\right), 7.55$ (d, 2H, Ar-H), 7.83 (d, 2H, Ar-H), 8.41 (bs, 2H, $\mathrm{NH}_{2}$ exchangeable with $\mathrm{D}_{2} \mathrm{O}$ ). Analysis Calcd for $\mathrm{C}_{20} \mathrm{H}_{14} \mathrm{~N}_{6} \mathrm{O}_{4} \mathrm{~S}$ (434.44); C, 55.30; H, 3.25; N, 19.34; S, 7.38\%. Found: C, 55.12; H, 3.01; N, 19.08; S, 7.24\%.

\section{References}

1. Ibrahim, M. A. Heterocycles 2011, 83, 2689. http://dx.doi.org/10.3987/REV-11-713 
2. Komarova, E. S.; Makarov, V. A.; Alekseeva, L. M.; Avamenko, G. V.; Granik, V. G.; Russ. Chem. Bull. 2007, 56, 2337. http://dx.doi.org/10.1007/s11172-007-0369-5

3. Blake, A. J.; Clarke, D.; Mares, R. W.; McNab, H. Org. Biomol. Chem. 2003, 1, 4268. http://dx.doi.org/10.1039/b306058f PMid:14685330

4. Katharkar, S. A.; Shinde, D. B. Bioorg. Med. Chem. Lett. 2006, 16, 6181. http://dx.doi.org/10.1016/j.bmcl.2006.09.040 PMid:17027265

5. Hassan, N. A. Molecules 2000, 5, 826. http://dx.doi.org/10.3390/50600826

6. Kolos, N. N.; Orlov, V. D.; Paponov, B. V.; Shishkin, O. V. Chem. Heterocycl. Compds. 1999, 35, 1207. http://dx.doi.org/10.1007/BF02323380

7. Joshi, K. C.; Dandia, A.; Khanna, S. Indian J. Chem. 1992, 31B, 105.

8. Zhang, G.; Chen, J. Lett. Org. Chem. 2011, 8, 180. http://dx.doi.org/10.2174/157017811795038377

9. Yamada, Y.; Yasuda, H.; Takayama, A. Heterocycles 1998, 48, 1185. http://dx.doi.org/10.3987/COM-98-8136

10. Hussein, A. H. M. Heteroatom Chem. 1997, 8, 1. http://dx.doi.org/10.1002/(SICI)1098-1071(1997)8:1<1::AID-HC1>3.0.CO;2-J

11. Reidl, Z.; Hajos, G.; Kover, P.; Kollenz, G. Arkivoc 2003, (v), 62. http://dx.doi.org/10.3998/ark.5550190.0004.506

12. Hajos, G.; Reidl, Z.; Gacs-Baitz, E.; Messmer, A. Tetrahedron 1992, 48, 8459. http://dx.doi.org/10.1016/S0040-4020(01)86594-7

13. Ibrahim, M. A.; Abdel-Rahman, R. M.; Abdel-Halim, A. M.; Ibrahim, S. S.; Allimony, H. A. J. Braz. Chem. Soc. 2009, 20, 1275. http://dx.doi.org/10.1590/S0103-50532009000700012

14. Ali, T.E.; Ibrahim, M.A. J. Braz. Chem. Soc. 2010, 21, 1007. http://dx.doi.org/10.1590/S0103-50532010000600010

15. Abdel-megid, M. Chem. Heterocycl. Compds. 2009, 45, 1523. http://dx.doi.org/10.1007/s10593-010-0460-y

16. Al-Najjar, A. A. A.; Amer, S. A.; Riad, M.; Elghamy, I.; Elnagdi, M. H. J. Chem. Res(S). 1996, 296.

17. Rohini, R.; Reddy, P. M.; Shanker, K.; Ravinder, V. Acta Chim. Slov. 2009, 56, 900.

18. Ibrahim, M.A.; Abdel-Rahman, R.M.; Abdel-Halim, A.M.; Ibrahim, S.S.; Allimony, H.A. Arkivoc 2008, (xvi), 202. http://dx.doi.org/10.3998/ark.5550190.0009.g19

19. Rashad, A. E.; Shamroukh, A. H.; Abdel-Megeid, R. E.; El-Sayed, W. A. Synth. Commun. 2010, 40, 1149. http://dx.doi.org/10.1080/00397910903050954 
20. El-Gazzar, A. B. A.; Aly, A. S.; Zaki, M. E. A.; Hafez, H. N. Phosphorus, Sulfur, Silicon 2008, 183, 2119.

21. Gross, D. C.; De Vay, S. E. Physiol. Plant Pathol. 1977, 11, 13.

22. Bauer, A. W.; Kirby, W. W. M.; Sherris, J. C.; Turck, M. Am. J. Clin. Pathol. 1966, 45, 493. PMid:5325707 